\title{
Appollonius Revisited: Supporting Spheres for Sundered Systems*
}

\author{
V. Klee, ${ }^{1}$ T. Lewis, ${ }^{2}$ and B. Von Hohenbalken ${ }^{3}$ \\ ${ }^{1}$ Department of Mathematics, University of Washington, \\ Box 354350, Seattle, WA 98195-4350, USA \\ klee@math.washington.edu \\ ${ }^{2}$ Department of Mathematics, University of Alberta, \\ Edmonton, Alberta, Canada T6G 2G1 \\ tlewis@vega.math.ualberta.ca \\ ${ }^{3}$ Department of Economics, University of Alberta, \\ Edmonton, Alberta, Canada T6G 2H4 \\ balder.von.hohenbalken@ualbert.ca
}

\begin{abstract}
When $C$ is a ball in $\mathbb{R}^{d}$ and $S$ is the sphere $\partial C$, we say that $S$ supports a convex body $B$ if $S$ intersects $B$ and either $B \subseteq C$ (then $S$ is a far support) or the interior of $C$ is disjoint from $B$ (then $S$ is a near support). The focus here is on common supports for a system $\mathcal{B}$ of $d+1$ bodies in $\mathbb{R}^{d}$ such that for each way of selecting a point from each member of $\mathcal{B}$, the selected points are affinely independent and hence form the vertex-set of a $d$-simplex. The main result asserts that if $\left(\mathcal{B}^{\prime}, \mathcal{B}^{\prime \prime}\right)$ is an arbitrary partition of $\mathcal{B}$, then there exists a unique Euclidean sphere that is simultaneously a near support for each member of $\mathcal{B}^{\prime}$ and a far support for each member of $\mathcal{B}^{\prime \prime}$.
\end{abstract}

\section{Introduction}

In all that follows, $\mathbb{R}^{d}$ denotes a real vector space of finite dimension $d$. A body is a nonempty compact convex subset of $\mathbb{R}^{d}$, and a proper body is one that is $d$-dimensional and hence has nonempty interior. We assume that a proper body $\mathbb{C}$ with $\mathbf{0} \in \operatorname{int}(\mathbb{C})$ is given, and we denote by $\|\cdot\|$ the norm that is the gauge function of $\mathbb{C}$; thus $\mathbb{C}=$ $\{x:\|x\| \leq 1\}$. This norm is such that $\|x+y\| \leq\|x\|+\|y\|$ and $\|\lambda x\|=\lambda\|x\|$ for all $x, y \in \mathbb{R}^{d}$ and $\lambda>0$. However, the equality $\|x\|=\|-x\|$ need not hold, for it is not assumed that $\mathbb{C}=-\mathbb{C}$.

A homothet of a set $X \subseteq \mathbb{R}^{d}$ is a set of the form $t+\lambda X$ for some $t \in \mathbb{R}^{d}$ and $\lambda>0$.

\footnotetext{
* The research of the first author was supported in part by the National Science Foundation.
} 
We use the term ball to denote a homothet of the unit ball $\mathbb{C}$ and the term sphere to denote a homothet of the unit sphere $\mathbb{S}=\{x:\|x\|=1\}$. Thus balls and spheres are sets of the forms $c+r \mathbb{C}=\{x:\|x-c\| \leq r\}$ and $c+r \mathbb{S}=\{x:\|x-c\|=r\}$, respectively, where $c \in \mathbb{R}^{d}$ and $r>0$. The point $c$ and the number $r$ are respectively the center and the radius of the ball and sphere. We are especially concerned with the situation in which spheres are Euclidean, meaning that $\mathbb{C}$ is the usual Euclidean ball centered at the origin. When this is assumed, the ambient space is denoted by $\mathbb{E}^{d}$ rather than $\mathbb{R}^{d}$.

When $C$ is a ball $c+r \mathbb{C}$ and $S$ is the sphere $c+r \mathbb{S}$, we say that $S$ supports a body $B$ if $S \cap B \neq \emptyset$ and either $B \subseteq C$ (then $S$ is a far support) or $B \cap \operatorname{int}(C)=\emptyset$ (then $S$ is a near support). These terms are motivated by the fact that $S$ is a near (resp. far) support for $B$ if and only if the intersection $S \cap B$ consists of all points of $B$ that are nearest to (resp. farthest from) the point $c$ with respect to the norm $\|\cdot\|$. (The points $b$ of $B$ that are nearest to $c$ are those for which $\|b-c\|$ is a minimum, and the points that are farthest from $c$ are those for which $\|b-c\|$ is a maximum.)

We are concerned with a system (finite collection) $\mathcal{B}$ of bodies in $\mathbb{R}^{d}$ and with the spheres that are common supports for all the bodies in the system. When $\left(\mathcal{B}^{\prime}, \mathcal{B}^{\prime \prime}\right)$ is a partition of a system $\mathcal{B}$, we call a sphere $S$ a $\mathcal{B}^{\prime}$-near $\mathcal{B}^{\prime \prime}$-far support for $\mathcal{B}$ provided that $S$ is a near support for each member of $\mathcal{B}^{\prime}$ and a far support for each member of $\mathcal{B}^{\prime \prime}$. When the members of $\mathcal{B}$ are indexed, this terminology is applied in the obvious way to partitions of the index-set.

We focus on systems $\mathcal{B}$ that are sundered, meaning that the members of the system are pairwise disjoint and, for each way of choosing a point from $d+1$ or fewer members of $\mathcal{B}$, the chosen points form the vertex-set of a simplex. (This is the same as being $(d-1)$-separated in the sense of [GPW]). Here is our main result:

Theorem 2.6. If spheres are Euclidean, then for each sundered system $\mathcal{B}=$ $\left(\mathcal{B}_{0}, \ldots, \mathcal{B}_{d}\right)$ of $d+1$ bodies in $\mathbb{E}^{d}$ and each partition $(I, J)$ of the index set, there is a unique I-near $J$-far supporting sphere for $\mathcal{B}$.

This theorem was inspired in part by the classical problem of Apollonius, which was concerned with constructing all supporting circles for a system of three circular disks in the plane. (For discussions of the problem of Apollonius, see [BM] and [CR]. See $[\mathrm{BFW}]$ and $[\mathrm{PW}]$ for recent studies of relatives and higher-dimensional analogues of this problem.) Our second inspiration was a recent theorem concerning common supporting hyperplanes for a sundered system of $d$ bodies. (That theorem was first stated by Bisztriczky [Bi], and first completely proved by Cappell et al. $\left[\mathrm{CGP}^{+}\right]$as part of a deeper and broader study. A simpler proof was given by the present authors [LVK].) In the present paper we have $d+1$ bodies and the supporting manifolds are spheres rather than hyperplanes. After the present paper was submitted, V. Soltan called our attention to a paper by Kramer and Németh [KN] in which the Brouwer fixed-point theorem is used to prove the special case of Theorem 2.6 in which $I=\{0, \ldots, d\}$, so that only near supports are involved.

Section 1 is devoted to a basic result (Theorem 1.1) whose proof is based on the Brouwer fixed-point theorem. This result guarantees the existence of supporting spheres for certain systems of $d+1$ bodies when the spheres are strictly convex. The assumption of strict convexity is removed in Corollary 1.4 by using stronger fixed-point theorems. In Section 2 special properties of Euclidean spheres are used to derive Theorem 2.6 from 
Theorem 1.1. Section 3 discusses the difficulties that arise in dealing with more general spheres when $d \geq 3$, and shows that when $d=2$, Theorem 2.6 remains valid as long as spheres are smooth and strictly convex.

\section{A Basic Theorem on Common Supporting Spheres}

A proper body (or the boundary of such a body) is strictly convex if the boundary does not contain any line segment. Of course, Euclidean balls and spheres have this property. Much that follows depends on the following basic result.

Theorem 1.1. Suppose that the balls in $\mathbb{R}^{d}$ are strictly convex, that $\left(B_{0}, \ldots, B_{d}\right)$ is a system of $d+1$ pairwise disjoint bodies in $\mathbb{R}^{d}$, and that, for each way of choosing a point from each $B_{k}$, there is a unique sphere that contains all the chosen points. Suppose that $(I, J)$ is a partition of $\{0, \ldots, d\}$, and that for each $j \in J$ the body $B_{j}$ is either a ball or a singleton. Then there is a sphere $S$ that is an I-near $J$-far support for the system $\left(B_{0}, \ldots, B_{d}\right)$.

Proof. For each point

$$
b=\left(b_{0}, \ldots, b_{d}\right) \in B=B_{0} \times \cdots \times B_{d}
$$

let $\gamma(b)$ denote the center of the unique sphere $S(b)$ that contains the set $\left\{b_{0}, \ldots, b_{d}\right\}$. Using the uniqueness of $\gamma(b)$ and the compactness of the sets $B_{k}$, a routine argument shows that the function

$$
\gamma: B \rightarrow \mathbb{R}^{d}
$$

is continuous and hence the image $\gamma(B)$ is a compact subset of $\mathbb{R}^{d}$.

Now we want to rule out the possibility that, for some $j \in J$, the set $\gamma(B)$ includes the center $c_{j}$ of $B_{j}$. Indeed, suppose that $b \in B$ with $\gamma(b)=c_{j}$, whence $S(b)$ is a sphere centered at $c_{j}$. However, then $S(b)$ misses $B_{j}$ if the radius of $S(b)$ exceeds that of $B_{j}$, while otherwise (since the sets $B_{k}$ are pairwise disjoint) $S(b)$ misses all the bodies $B_{k}$ for $k \neq j$.

Now we assume temporarily that the norm is strictly convex, and we define a singlevalued mapping

$$
\sigma_{(I, J)}: \gamma(B) \rightarrow B
$$

by setting (for each $w \in \gamma(B)$ )

$$
\sigma_{(I, J)}(w)=\left(p_{0}, \ldots, p_{d}\right) \in B,
$$

where, for each $i \in I, p_{i}$ is the unique point $q \in B_{i}$ for which the distance $\|q-w\|$ is a minimum, and, for each $j \in J, p_{j}$ is the unique point $q \in B_{j}$ for which the distance $\|q-w\|$ is a maximum. (Uniqueness of $q$ follows from strict convexity of the norm.)

The continuity of the mapping $\sigma_{(I, J)}$ follows from a routine argument using the compactness of the sets $B_{k}$, the uniqueness conditions, and the continuity properties of the norm. Hence the composed mapping

$$
\sigma_{(I, J)} \circ \gamma: B \rightarrow B
$$


is continuous. Since $B$ is a compact convex set, Brouwer's fixed-point theorem implies the existence of a point $b \in B$ such that $b=\sigma_{(I, J)}(\gamma(b))$. However, it is then evident that the sphere $S(b)$ is the desired $I$-near $J$-far support for the system $\left(B_{0}, \ldots, B_{d}\right)$. That completes the proof.

The reader who is interested only in support by Euclidean spheres may skip the rest of this section, which shows that Theorem 1.1 is valid even when balls are not strictly convex. First, we need two lemmas.

Lemma 1.2. Suppose that $0<\tau<1$, and that $B$ is a proper body with $\mathbf{0} \in \partial B$. Then the intersection $(\partial B) \cap(\tau B)$ is starshaped from the point $\mathbf{0}$.

Proof. Consider an arbitrary point $p \in((\partial B) \cap(\tau B)) \backslash\{\mathbf{0}\}$. Since $(1 / \tau) p \in B$, there exists $\mu \geq 1 / \tau$ such that $\mu p \in \partial B$. However, then the collinear points $\mathbf{0}, p$, and $\mu p$ all belong to $\partial B$, whence (by $B$ 's convexity) the segment $[\mathbf{0}, \mu p]$ is contained in $\partial B$. Hence $[\mathbf{0}, p] \subseteq(\partial B) \cap(\tau B)$ and it follows that $(\partial B) \cap(\tau B)$ is starshaped from $\mathbf{0}$.

Lemma 1.3. For the unit ball $\mathbb{C}$ of $\mathbb{R}^{d}$, and for $w \in \mathbb{R}^{d} \backslash\{\mathbf{0}\}$, set

$\delta=\max \{\|x-w\|: x \in \mathbb{C}\}, \quad Y=\{y \in \mathbb{C}:\|y-w\|=\delta\}, \quad y=\frac{1}{\|-w\|}(-w)$.

Then $\delta=1+\|-w\|, y \in Y$, and the set $Y$ is compact and starshaped from $y$. If the norm $\|\cdot\|$ is strictly convex, then $Y=\{y\}$.

Proof. For each $x \in \mathbb{C}$ we have

$$
\begin{aligned}
\|x-w\| & \leq\|x\|+\|-w\| \leq 1+\|-w\| \\
& =\left(\frac{1}{\|-w\|}+1\right)\|-w\|=\left\|\frac{1}{\|-w\|}(-w)-w\right\|=\|y-w\| .
\end{aligned}
$$

It follows from this that $\delta=1+\|-w\|$ and $y \in Y$.

That $Y$ is compact follows from a routine argument based on continuity of the norm. To see that $Y$ is starshaped from $y$, apply Lemma 1.2 with $B=C-y$, after verifying that

$$
\{z:\|z\|<1\}-y=\frac{1}{1+\|-w\|}(\{z:\|z-w\| \leq 1+\|-w\|\}-y) .
$$

Corollary 1.4. Theorem 1.1 is valid without the assumption of strict convexity.

Proof. We need a more general fixed-point theorem because the function $\sigma_{(I, J)}$ may no longer be single-valued. However, it is easy to verify that $\sigma_{(I, J)}$ is an upper-semicontinuous set-valued function, so the same is true of the composed mapping

$$
\sigma_{(I, J)} \circ \gamma: B \rightarrow 2^{B}
$$


where $2^{B}$ denotes the space of all nonempty compact subsets of $B$. For each $w \in \gamma(B)$, the point $p_{i}$ defined earlier now becomes a compact convex subset $P_{i}$ of the body $B_{i}$, and the point $p_{j}$ becomes a starshaped (and hence contractible) compact subset $P_{j}$ of the body $B_{j}$. (To verify starshapedness, use Lemma 1.3.) The set $\sigma_{(I, J)}(w)$ is the product of the $P_{i}$ 's and the $P_{j}$ 's and is therefore a compact contractible subset of the product $B$. It now follows from the fixed-point theorems of Eilenberg and Montgomery [EM] and Begle [Be] that $b \in \sigma_{(I, J)}(\gamma(b))$ for some $b \in B$. (When $J$ is empty, the set $\sigma_{(I, J)}(w)$ is always convex and we could instead use the more elementary fixed-point theorem of Kakutani $[\mathrm{K}]$.) As before, the sphere $S(b)$ is the desired common support.

Now we want to find geometrically interesting circumstances under which the conditions of Theorem 1.1 are satisfied, we want to deal with systems of arbitrary bodies rather than requiring that some of the bodies are singletons or balls, and we want to address the uniqueness of the $I$-near $J$-far support. We are able in Section 2 to achieve all of these goals for the case in which spheres are Euclidean, and in Section 3 for the case in which $d=2$ and spheres are smooth and strictly convex.

\section{Euclidean Spheres Supporting Sundered Systems of $d+1$ Bodies}

The basic assumption in Theorem 1.1 is that for each way of choosing a point from each of the sets $B_{0}, \ldots, B_{d}$, there is a unique sphere containing the chosen points. As is evident from the following remark, this condition is satisfied when spheres are Euclidean and the system $\left(B_{0}, \ldots, B_{d}\right)$ is sundered.

Lemma 2.1. For each affinely independent set $V$ of $d+1$ points in $\mathbb{E}^{d}$, there is a unique Euclidean sphere that contains $V$.

Proof. Let $V=\left\{v_{0}, \ldots, v_{d}\right\}$, and assume without loss of generality that $v_{0}=\mathbf{0}$. Note that a point $c$ is the center of a sphere containing $V$ if and only if $\left\langle c-v_{0}, c-v_{0}\right\rangle=$ $\left\langle c-v_{k}, c-v_{k}\right\rangle$ for all $k$, where $\langle\cdot, \cdot\rangle$ is the usual inner product. With $v_{0}=\mathbf{0}$, this is equivalent to the condition that $\left\langle c, v_{k}\right\rangle=\left\langle v_{k}, v_{k}\right\rangle / 2$ for all $k$. Since the set $\left\{v_{1}, \ldots, v_{d}\right\}$ is linearly independent, there is a unique solution $c$.

We have not yet addressed the uniqueness of the $I$-near $J$-far supporting sphere for a given $(I, J)$. That is done in the following two results about arbitrary bodies.

Lemma 2.2. Suppose that $B$ is a body in $\mathbb{E}^{d}$, and $C$ and $D$ are distinct Euclidean balls whose bounding spheres $S=\partial C$ and $T=\partial D$ are both near supports or both far supports of $B$. Let $W=S \cap T$. Then precisely one of the following statements is correct:

(a) $C \subset D$ or $D \subset C$, and $W$ consists of a single point of $B$.

(b) $S$ and $T$ are near supports that are separated by a hyperplane (whence $W$ is empty or a singleton) and each separating hyperplane $H$ intersects $B$.

(c) The affine hull of $W$ is a hyperplane $H$ that intersects $B$. 
Proof. When $C \subset D$, it is clear from the "distinct" and the "support" hypotheses that $C$ has smaller radius but $C$ is not contained in int $(D)$. Hence $W$ consists of a single point, and that point belongs to $B$ since both $S$ and $T$ intersect $B$. The same argument applies when $D \subset C$, so for the rest of this proof we may assume that neither ball contains the other.

Each hyperplane $H$ that separates $S$ and $T$ must be intersected by $B$, for $B$ is convex and intersects both $S$ and $T$. If there is such an $H$, and $S$ and $T$ are both far supports of $B$, it follows (using the convexity of $B$ and the strict convexity of Euclidean balls) that the sets $S \cap B, T \cap B$, and $B$ are all equal to the same singleton, whence $S$ and $T$ are also near supports of $B$. That disposes of (b).

There remains the case in which the sets $\operatorname{int}(C)$ and $\operatorname{int}(D)$ intersect but neither is contained in the other. Then (using a basic property of Euclidean balls) $W$ is contained in a hyperplane $H$. With $H_{+}$and $H_{-}$denoting the two open half-spaces bounded by $H$, the notation may be chosen so that $D \cap H_{+} \subset C$ and $C \cap H_{-} \subset D$. Note that if points $x$ and $y$ of $B$ are near support points for $S$ and $T$, respectively, then $x$ lies in $H_{+}$and $y$ lies in $H_{-}$. The reason is that if (for example) $x$ were not in $H_{+}$, it would have to be in $\operatorname{int}(D)$, which implies that $y$ could not be a near support point for $T$. Thus $B$ contains points in both half-spaces $H_{+}$and $H_{-}$and hence (since $B$ is convex) $B$ meets $H$. A similar argument applies when $x$ and $y$ are far support points for $C$ and $D$, respectively.

Theorem 2.3. Suppose that $\mathcal{B}=\left(B_{0}, \ldots, B_{d}\right)$ is a sundered system of +1 bodies in $\mathbb{E}^{d}$, and $(I, J)$ is a partition of the index-set. Then at most one Euclidean sphere is an $I$-near $J$-far support for $\mathcal{B}$.

Proof. Suppose that there are two distinct spheres, $S$ and $T$, of the mentioned sort, and let $W=S \cap T$. For each index $k, S$ and $T$ must be related to $B_{k}$ in one of the manners (a), (b), and (c) described in Lemma 2.2.

Suppose first that the situation (a) arises for $k=m$, with (say) $C \subset D$, and note that then $C \backslash W \subset \operatorname{int}(D)$. Consider an arbitrary index $n \neq m$. Since the bodies $B_{k}$ are pairwise disjoint, $B_{n}$ misses $W$. However, then $S$ must miss $B_{n}$ if $T$ is a near support of $B_{n}$, and $T$ must miss $B_{n}$ if $S$ is a far support of $B_{n}$. This shows that (a) cannot occur for any $B_{k}$, so (b) and (c) are the only possibilities.

For each index $k$, let the points $a_{k}$ and $b_{k}$ belong to $B_{k} \cap S$ and $B_{k} \cap T$, respectively. If condition (b) or condition (c) is satisfied and the hyperplane $H$ is as described there, then $H$ intersects the segment $\left[a_{k}, b_{k}\right]$ and hence (by convexity) intersects each set $B_{k}$. However, this contradicts the assumption that the system $B$ is sundered.

Corollary 2.4. Suppose that spheres are Euclidean, that $\left(B_{0}, \ldots, B_{d}\right)$ is a sundered system of $d+1$ bodies in $\mathbb{E}^{d}$, that $(I, J)$ is a partition of $\{0, \ldots, d\}$, and that for each $j \in J$ the body $B_{j}$ is either a ball or a singleton. Then there is a unique sphere $S$ that is an I-near $J$-far support for the system $\left(B_{0}, \ldots, B_{d}\right)$.

Proof. Use Lemma 2.1 and Theorem 2.3 in conjunction with Theorem 1.1. 
To complete the discussion of common supports for sundered systems of $d+1$ bodies when spheres are Euclidean, we want to permit all the bodies in Corollary 2.4 to be arbitrary, rather than insisting that some of them are balls or singletons. The proof that this is possible will be based on an induction involving the assertions $A(d, f)$ and $A_{0}(d, f)$, where these are defined as follows for $0 \leq f \leq d+1$ :

$A(d, f)$ is the assertion that whenever $\mathcal{B}=\left(B_{0}, \ldots, B_{d}\right)$ is a sundered system of $d+1$ bodies in $\mathbb{E}^{d}$ and $(I, J)$ is a partition of the index-set with $|J|=f$, there exists an $I$-near $J$-far supporting sphere for $\mathcal{B}$.

$A_{0}(d, f)$ is the restriction of $A(d, f)$ to the case in which at least one member of $\mathcal{B}$ is a singleton.

Note that by Corollary 2.4, $A(d, 0)$ holds for all $d$.

Lemma 2.5. Suppose that $0 \leq f \leq d+1$. If the assertion $A_{0}(d+1, f)$ is true, then $A(d, f)$ is also true.

Proof. Suppose that $\mathcal{B}=\left(B_{0}, \ldots, B_{d}\right)$ is a sundered system of $d+1$ bodies in $\mathbb{E}^{d}$, and $(I, J)$ is a partition of $\{0, \ldots, d\}$ with $|J|=f$. Let $\mathbb{E}^{d}$ be embedded in the usual way in $\mathbb{E}^{d+1}$ and consider the system $\mathcal{B}^{\prime}=\left(B_{0}, \ldots, B_{d}, B_{d+1}\right)$ in $\mathbb{E}^{d+1}$, where $B_{d+1}$ is a singleton in $\mathbb{E}^{d+1} \backslash \mathbb{E}^{d}$. Let $I^{\prime}=I \cup\{d+1\}$. It follows from $A_{0}(d+1, f)$ that $\mathbb{E}^{d+1}$ contains an $I^{\prime}$-near $J$-far supporting sphere $S$ for the system $\mathcal{B}^{\prime}$. However, then the sphere $S \cap \mathbb{E}^{d}$ is an $I$-near $J$-far supporting sphere for the system $\mathcal{B}$.

The following is the main result of this paper.

Theorem 2.6. If spheres are Euclidean, then for each sundered system $\mathcal{B}=$ $\left(\mathcal{B}_{0}, \ldots, \mathcal{B}_{d}\right)$ of $d+1$ bodies in $\mathbb{E}^{d}$ and each partition $(I, J)$ of $\{0, \ldots, d\}$, there exists a unique I-near $J$-far supporting sphere for $\mathcal{B}$.

Proof. It suffices to establish the existence of an $I$-near $J$-far supporting sphere, for uniqueness then follows from Theorem 2.3. In view of Lemma 2.5, it suffices to prove, for each $d$, that $A_{0}(d, f)$ holds for all relevant $f$. The assertion $A_{0}(d, 0)$ has already been proved. Supposing that $0<f \leq d+1$ and that $A_{0}(d, f-1)$ is known to hold, we want to prove $A_{0}(d, f)$. In taking this inductive step, we assume the notation chosen so that $B_{0}$ is the singleton $\{\boldsymbol{0}\}, I=\{0, \ldots, d-f\}$, and $J=\{d-f+1, \ldots, d\}$.

Let $I_{+d}=I \cup\{d\}$ and $J_{-d}=J \backslash\{d\}$. By a theorem of Bisztriczky [Bi] and others $\left[\mathrm{CGP}^{+}\right],[\mathrm{LVK}]$, the sundered system $\left(B_{0}, \ldots, B_{d-1}\right)$ admits exactly two $\left(I, J_{-d}\right)$ supporting hyperplanes $H$ and $P$. We assume that the intersection $H \cap P$ is a $(d-2)$-flat $G$, for in the remaining case $H$ and $P$ are parallel and a similar argument is applicable.

The complement of $H \cup P$ has four open components and these are symmetric in pairs with respect to the flat $G$. In one of these pairs, neither of the components intersects the union $\bigcup_{k<d} B_{k}$. In the other pair, the closure of one component contains $\bigcup_{i \in I} B_{i}$ and the closure of the other component contains $\bigcup_{j \in J_{-d}} B_{j}$. Letting $H^{+}$and $H^{-}$denote the two open half-spaces bounded by $H$ and letting $P^{+}$and $P^{-}$denote the two open half-spaces 
bounded by $P$, we may assume the notation chosen so that $\bigcup_{i \in I} B_{i} \subset \operatorname{cl}\left(H^{-} \cap P^{+}\right)$and $\bigcup_{j \in J_{-d}} B_{j} \subset \operatorname{cl}\left(H^{+} \cap P^{-}\right)$.

From the fact that the family $\left\{B_{0}, \ldots, B_{d}\right\}$ is sundered and $B_{d}$ is convex, it follows that the set $B_{d}$ is contained in the intersection $H^{+} \cap P^{+}$or in the intersection $H^{-} \cap P^{-}$. We assume without loss of generality that $B_{d} \subset H^{+} \cap P^{+}$.

Since $B_{0}=\{\boldsymbol{0}\}$, every transversal hyperplane of the system $\left(B_{0}, \ldots, B_{d-1}\right)$ passes through the origin. From this it follows that the union $Y$ of all such hyperplanes is starshaped from $\mathbf{0}$, and hence that each component of $\mathbb{E}^{d} \backslash Y$ is an open connected union of open rays issuing from $\mathbf{0}$.

Choose an arbitrary point $q$ of $B_{d}$. For each $\tau \geq 1$, the inductive hypothesis guarantees the existence of a unique ball $C(\tau)$ such that the sphere $S(\tau)=\partial C(\tau)$ is an $I_{+d}$-near $J_{-d^{-}}$ far support of the system $\left(B_{0}, \ldots, B_{d-1},\{\tau q\}\right)$. (Since $\{\tau q\}$ is merely a singleton, $S(\tau)$ is also an $I$-near $J$-far support of this system.) A routine argument based on uniqueness and compactness establishes the continuity (with respect to the Hausdorff-metric for the images) of the set-valued mapping that sends $\tau \geq 1$ into the ball $C(\tau)$. We show in the next paragraph that, for some sufficiently large $\tau, B_{d} \subseteq C(\tau)$. It then follows from the continuity of $C(\cdot)$ that there is a smallest $\tau_{0} \geq 1$ such that $B_{d} \subseteq C\left(\tau_{0}\right)$, and the sphere $S\left(\tau_{0}\right)=\partial C\left(\tau_{0}\right)$ is then the desired $I$-near $J$-far supporting sphere for the original system $\left(B_{0}, \ldots, B_{d}\right)$. Again, the uniqueness follows from Theorem 2.3.

For each positive integer $m$ and for $0 \leq k<d$, let $b_{k}^{m}$ be a point of the intersection $S(m) \cap B_{k}$. Since the system $\left(B_{0}, \ldots, B_{d-1}\right)$ is sundered, the set $\left\{b_{0}^{m}, \ldots, b_{d-1}^{m}\right\}$ determines a hyperplane $H(\mathrm{~m})$ and there is a unique point $a(\mathrm{~m})$ of $H(\mathrm{~m})$ that is equidistant from all the points $b_{0}^{m}, \ldots, b_{d-1}^{m}$. The center $c(m)$ of $S(m)$ lies on the line that passes through $a(m)$ and is orthogonal to $H(m)$. Note also that $\|c(m)\| \rightarrow \infty$ as $m \rightarrow \infty$, for both the origin and the point $m q$ belong to $S(m)$. Let

$$
u(m)=\frac{1}{\|c(m)-a(m)\|}[c(m)-a(m)] .
$$

By compactness, there is a sequence $m_{1}<m_{2}<\cdots$, such that the sequence $u\left(m_{1}\right), u\left(m_{2}\right), \ldots$, converges to a point $u$ of unit norm, and, for each $k$, the sequence $b_{k}^{m_{1}}, b_{k}^{m_{2}}, \ldots$, converges to a point $b_{k} \in B_{k}$. (Note that $b_{0}=\mathbf{0}$.) In conjunction with the smoothness of Euclidean balls, this guarantees that the sequence $C\left(m_{1}\right), C\left(m_{2}\right), \ldots$, converges to a closed half-space $H^{\prime}$ whose bounding hyperplane passes through the origin and has $u$ as inner normal. (This convergence is in the sequential set-theoretic sense described in [W].) Since $B_{d}$ is compact and is interior to $H^{\prime}$, it follows that $B_{d} \subseteq C\left(m_{t}\right)$ for large enough $t$. That completes the proof.

Corollary 2.7. If $\left\{B_{0}, \ldots, B_{d}\right\}$ is a sundered family of $d+1$ bodies in $\mathbb{E}^{d}$, the number of common supporting spheres is precisely $2^{m}$, where $m$ is the number of bodies that are not singletons.

Proof. Use the fact that if a body $B$ is not a singleton, no Euclidean sphere can be simultaneously a near support and a far support for $B$.

The following result might be called a "near-converse" of Theorem 2.6. 
Theorem 2.8. Suppose that $\mathcal{B}=\left(B_{0}, \ldots, B_{d}\right)$ is a system of $d+1$ bodies in $\mathbb{E}^{d}$, and that for each partition $(I, J)$ of $\{0, \ldots, d\}$ there exists a sphere that is an I-near $J$-far support for $\mathcal{B}$. Suppose also that no member of $\mathcal{B}$ is a singleton. Then no hyperplane intersects the relative interior of each member of $B$.

Proof. Suppose that the conclusion fails, and let $P$ be a hyperplane which for each $k$ intersects the relative interior $\operatorname{rint}\left(B_{k}\right)$. For each $k$, choose $b_{k} \in P \cap \operatorname{rint}\left(B_{k}\right)$. It follows from a lemma of Radon $[\mathrm{R}]$ that there is a partition $(I, J)$ of $\{0, \ldots, d\}$ such that

$$
\operatorname{conv}\left\{b_{i}: i \in I\right\} \cap \operatorname{conv}\left\{b_{j}: j \in J\right\} \neq \emptyset .
$$

By hypothesis, there exists a sphere $S$ that is an $I$-near $J$-far supporting sphere for $\mathcal{B}$, and there exists a sphere $T$ that is an $I$-far $J$-near support for $\mathcal{B}$. Since at least one member of $\mathcal{B}$ is not a singleton, $S$ and $T$ cannot coincide. In fact, the intersection $S \cap T$ must be a $(d-2)$-sphere $W$ that lies in a hyperplane $H$. Let $C$ and $D$ denote the balls that are bounded by $S$ and $T$, respectively. Then

$$
B_{i} \subseteq D \backslash \operatorname{int}(C) \quad \text { for all } i \in I \quad \text { and } \quad B_{j} \in C \backslash \operatorname{int}(D) \quad \text { for all } j \in J,
$$

whence $\bigcup_{i \in I} \operatorname{int}\left(B_{i}\right)$ lies in one of the two closed half-spaces bounded by $H$ and $\bigcup_{j \in J} B_{j}$ lies in the other such closed half-space. Note also that

$$
(D \backslash \operatorname{int}(C)) \cap H=W=(C \backslash \operatorname{int}(D)) \cap H,
$$

and that $W$ does not contain any segment. Since no $B_{k}$ is a singleton and since always $b \in \operatorname{rint}\left(B_{k}\right)$, it follows that the sets $\left\{b_{i}: i \in I\right\}$ and $\left\{b_{j}: j \in J\right\}$ lie in the opposite open half-spaces bounded by $H$, contradicting the fact that their convex hulls intersect.

\section{Non-Euclidean Supporting Spheres}

Now we abandon the assumption that spheres are Euclidean, thus reverting to the situation in Section 1.

A proper body $C$ (or its boundary $\partial C$ ) is smooth if there is a unique supporting hyperplane at each boundary point. It was proved by Kalisch and Straus [KS] and by Gromov [Gr] that if $C$ is smooth and $T$ is a $d$-simplex, then some positive homothet of $T$ is inscribed in $\partial C$; Gromov stated also that this property characterizes smoothness. Since inscribing $T$ in some homothet of $\partial C$ is equivalent to inscribing some homothet of $T$ in $\partial C$, we may state the following:

Theorem 3.1 [KS], [Gr]. A proper body $C$ in $\mathbb{R}^{d}$ is smooth if and only if each $d$-simplex in $\mathbb{R}^{d}$ is inscribed in some positive homothet of $C$ 's boundary.

Recall that our basic Theorem 1.1 on common supports used not only the existence but also the uniqueness of the sphere containing the vertex-set of a given $d$-simplex. Although Theorem 3.1 takes care of the existence whenever spheres are smooth, the following striking result of Shaǐdenko [S] and Goodey [Go] shows that uniqueness does not apply when $d \geq 3$ and the spheres are not Euclidean. 
Theorem 3.2 [S], [Go]. Suppose that $\geq 3$, and $K$ and $K^{\prime}$ are proper convex bodies in $\mathbb{R}^{d}$ such that the intersection of $K$ with any translate of $K^{\prime}$ is contained in a hyperplane. Then $K$ and $K^{\prime}$ are homothetic ellipsoids.

Theorem 3.2 implies that when $d \geq 3$ and the unit sphere $\mathbb{S}$ is not an ellipsoid, there is a translate $S^{\prime}$ of $\mathbb{S}$ such that $\mathbb{S} \cap S^{\prime}$ is not contained in any hyperplane. However, then $\mathbb{S} \cap S^{\prime}$ contains the vertex-set $V$ of some $d$-simplex, and $\mathbb{S}$ itself contains two translates of $V$.

The facts are kinder when $d=2$, as was noted (in different language) by Kalisch and Straus [KS]:

Theorem 3.3 [KS]. When $d=2$, the following two conditions on spheres are equivalent:

(a) Spheres are strictly convex.

(b) Each triangle admits at most one circumscribing sphere.

Proof. The following condition is equivalent to (b):

(c) For each triangle $\mathrm{T}$, at most one positive homothet of $\mathrm{T}$ is inscribed in the unit sphere $\mathbb{S}$.

Hence it suffices to show that (a) and (c) are equivalent. In doing this, we use $p q$ to denote the Euclidean distance between points $p$ and $q$ and we use $[p, q, r]$ to denote the triangle having vertices $p, q$, and $r$.

Suppose that $\mathbb{S}$ contains a segment $[v, z]$, let the points $v, w, x, y, z$ be equally spaced along $[v, z]$ (i.e., $v w=w x=x y=y z$ ), and let $w^{\prime}$ and $y^{\prime}$ denote the points of $\mathbb{S}$ such that the segments $\left[w, w^{\prime}\right]$ and $\left[y, y^{\prime}\right]$ are both perpendicular to $[v, z]$. We assume without loss of generality that $w w^{\prime} \leq y y^{\prime}$. Then there are points $v^{\prime} \in[v, w]$ and $x^{\prime} \in[w, x]$ such that $v^{\prime} w=w x^{\prime}$ and $v^{\prime} x^{\prime} / w w^{\prime}=x z / y y^{\prime}$. The isosceles triangles $\left[v^{\prime}, w^{\prime}, x^{\prime}\right]$ and $\left[x, y^{\prime}, z\right]$ are homothetic and both are inscribed in $\mathbb{S}$.

To show that (a) implies (c), we assume that $\mathbb{S}$ is strictly convex and yet there are two distinct homothetic triangles inscribed in $\mathbb{S}$. Let the triangles be $[a, b, c]$ and $\left[a^{\prime}, b^{\prime}, c^{\prime}\right]$, with homothety center $e$. (If the triangles are translates, a similar argument applies). No two sides can be collinear, so we may assume that the ray $[e, b]$ lies between the rays $[e, a]$ and $[e, c]$. However, then either $b$ is interior to $\left[a, c, b^{\prime}\right]$, or $b^{\prime}$ is interior to $\left[a^{\prime}, c^{\prime}, b\right]$.

Theorem 3.4. Suppose that $d=2$ and spheres are smooth. Let $\mathcal{B}$ be a sundered system $\left(B_{0}, B_{1}, B_{2}\right)$ of three bodies in $\mathbb{R}^{d}$, and let $(I, J)$ be a partition of $\{0,1,2\}$. Then there exists an I-near $J$-far supporting sphere for $\mathcal{B}$. When spheres are strictly convex as well as smooth, this I-near J-far supporting sphere is unique.

Proof. To deal with spheres that are both smooth and strictly convex, use Theorems 1.1, 3.1, and 3.3. Then pass by approximation to the case in which spheres are merely smooth. 
The problem of the existence of common supporting spheres is still open in the smooth non-Euclidean case when $d \geq 3$. For that case, we have neither results nor counterexamples.

\section{References}

[Be] E. G. Begle, A fixed-point theorem, Ann. Math. 51 (1950), 544-550.

[Bi] T. Bisztriczky, On separated families of convex bodies, Arch. Math. 54 (1990), 193-199.

[BM] C. B. Boyer and U. C. Merzbach, A History of Mathematics, Wiley, New York, 1991.

[BFW] A. Bruen, J. C. Fisher, and J. B. Wilker, Apollonius by inversion, Math. Mag. 56 (1983), 97-103.

$\left[\mathrm{CGP}^{+}\right]$S. E. Cappell, J. E. Goodman, J. Pach, R. Pollack, M. Sharir, and R. Wenger, Common tangents and common transversals, Adv. in Math. 106 (1994), 198-215.

[CR] R. Courant and H. Robbins, What is Mathematics?, Oxford University Press, London, 1941.

[EM] S. Eilenberg and D. Montgomery, Fixed-point theorem for multivalued transformations, Amer. J. Math. 68 (1946), 214-222.

[Go] P. R. Goodey, Homothetic ellipsoids, Math. Proc. Cambridge Philos. Soc. 93 (1983), 25-34.

[GPW] J. E. Goodman, R. Pollack, and R. Wenger, Geometric transversal theory, in New Trends in Discrete and Computational Geometry (J. Pach, ed.), Springer-Verlag, Berlin, 1993, pp. 163-198.

[Gr] M. L. Gromov, Simplexes inscribed on a hypersurface (Russian), Mat. Zametki 5 (1969), 81-89. (Transl. Math. Notes 5 (1969), 52-56.)

[K] S. Kakutani, A generalization of Brouwer's fixed-point theorem, Duke Math. J. 8 (1941), 457-459.

[KS] G. K. Kalisch and E. G. Straus, On the determination of points in a Banach space by their distances from points of a given set, Acad. Brasil. Ci. 29 (1958), 501-519.

[KN] H. Kramer and A. B. Németh, Supporting spheres for families of independent convex sets, Arch. Math. 24 (1973), 91-96.

[LVK] T. Lewis, B. Von Hohenbalken, and V. Klee, Common supports as fixed points, Geom. Dedicata 60 (1996), 277-281.

[PW] M. Paluzny and J. B. Wilker, A case of the three-dimensional problem of Apollonius, Aequationes Math. 41 (1991), 172-186.

[R] J. Radon, Mengen konvexer Körper, die einen gemeinsamen Punkt enthalten, Math. Ann. 83 (1921), $113-115$.

[S] A. V. Shaǐdenko, Some characteristic properties of the ellipsoid (in Russian), Sibirsk Mat. Zh. 21 (1980), 232-234, 240.

[W] G. T. Whyburn, Analytic Topology, American Mathematical Society Colloquium Publications, Vol. 27, American Mathematical Society, Providence, RI, 1942.

Received August 2, 1995, and in revised form November 30, 1995. 